\title{
Binding free energy, energy and entropy calculations using simple model systems
}

\author{
Balder Lai $\cdot$ Chris Oostenbrink
}

Received: 10 July 2012/ Accepted: 25 August 2012/Published online: 23 September 2012

(C) The Author(s) 2012. This article is published with open access at Springerlink.com

\begin{abstract}
Free energy differences are calculated for a set of two model host molecules, binding acetone and methanol. Two active sites of different characteristics were constructed based on an artificially extended C60 fullerene molecule, possibly functionalised to include polar interactions in an otherwise apolar, spherical cavity. The model host systems minimise the necessary sampling of conformational space while still capturing key aspects of ligand binding. The estimates of the free energies are split up into energetic and entropic contributions, using three different approaches investigating the convergence behaviour. For these systems, a direct calculation of the total energy and entropy is more efficient than calculating the entropy from the temperature dependence of the free energy or from a direct thermodynamic integration formulation. Furthermore, the compensating surrounding-surrounding energies and entropies are split off by calculating reduced ligandsurrounding energies and entropies. These converge much more readily and lead to properties that are more straightforwardly interpreted in terms of molecular interactions and configurations. Even though not experimentally accessible, the reduced thermodynamic properties may prove highly relevant for computational drug design, as they may give direct insights into possibilities to further optimise ligand binding while optimisation in the surrounding-surrounding
\end{abstract}

Electronic supplementary material The online version of this article (doi:10.1007/s00214-012-1272-1) contains supplementary material, which is available to authorized users.

B. Lai · C. Oostenbrink $(\square)$

Institute of Molecular Modeling and Simulation,

University of Natural Resources and Life Science Vienna,

Muthgasse 18, Vienna, Austria

e-mail: chris.oostenbrink@boku.ac.at energy or entropy will exactly cancel and not lead to improved affinity.

Keywords Entropy $\cdot$ Enthalpy $\cdot$ Ligand-surrounding energy · Fullerene model system · Thermodynamic integration - Thermodynamics

\section{Introduction}

Drug design (DD) often requires the binding affinity optimisation of lead compounds or known drugs, which is commonly achieved by the substitution of atoms or groups of atoms in the molecule or by restricting the conformational freedom of the molecules. These modifications should not affect the pharmacophoric features or the interactions with the binding pocket negatively in a significant way, but rather increase binding affinity, that is, induce a favourable change in binding free enthalpy $\left(\Delta G_{\text {bind }}\right)$. Therefore, improving $\Delta G_{\text {bind }}$ forms the main focus during the optimisation process. However, retrospective analyses [1] have shown that a rational modification often only leads to a moderate improvement in $\Delta G_{\text {bind }}$, due to a compensation of the enthalpy $(\Delta H)$ and the entropy $(\Delta S)$. A shift from mainly entropically driven binding towards mainly enthalpically driven binding, or vice versa, is commonly observed [2-4]. This phenomenon is largely due to the current trend to optimise ligands for more enthalpic binding. It is common that the entropic contribution is dominant for a compound that is the first of its class, while further optimisations lead to stronger enthalpic binding in the best of its class [5].

In the last few years, experimental approaches that attempt to take into account all three mentioned thermodynamic terms, $\Delta G, \Delta H$ and $\Delta S$, have gained popularity 
$[6,7]$. Examples are isothermal titration calorimetry (ITC) and surface plasmon resonance (SPR) techniques. These methods offer valuable insight into the effect of modifications in the molecular structure on the affinity and help to adjust design strategies in directions that improve either the enthalpic or the entropic contributions. An accurate estimation of $\Delta G, \Delta H$ and $\Delta S$, by computational means allows for focus of the design on $\Delta H$ or $\Delta S$, depending on the specific aims for the ligand. However, estimating $\Delta H$ and $\Delta S$ in silico still proves to be a challenge and computationally expensive [8, 9]. Simplified host-guest systems offer an attractive tool to assess the accuracy and efficiency of free energy calculations $[10,11]$.

Here, an attempt is made to estimate $\Delta G, \Delta H$ and $\Delta S$ of binding for two simple host models previously used to illustrate the efficiency of free energy methods [12]. The two host models, as shown in Fig. 1, are C60 fullerenes with carbon-carbon bonds extended to $0.2 \mathrm{~nm}$, which can be considered to be representative for a mostly rigid hydrophobic binding pocket. The only difference between the first $\left(\mathrm{C}_{\mathrm{APO}}\right)$ and second $\left(\mathrm{C}_{\mathrm{HB}}\right)$ host model is that the latter has an acetamide group, $-(\mathrm{C}=\mathrm{O}) \mathrm{NH}_{2}$, attached to one of the carbon atoms which introduces hydrogen bond forming capabilities. Correspondingly simple ligands, acetone and methanol, were chosen and kept inside the host models in all simulations. These simplifications result in a minimal computational system that allows for faster convergence of molecular interactions and characterisation of various methods to estimate enthalpic and entropic effects.

A popular approach to estimate entropic contributions to ligand binding is through the calculation of configurational entropies through heuristic [13] or quasi harmonic analysis [14] or variations thereof [15-17]. Relevant interpretations of experimental data have been possible with this approach $[18,19]$. However, it is clear that entropic contributions due to the solvent may play significant roles [20]. Ideally,

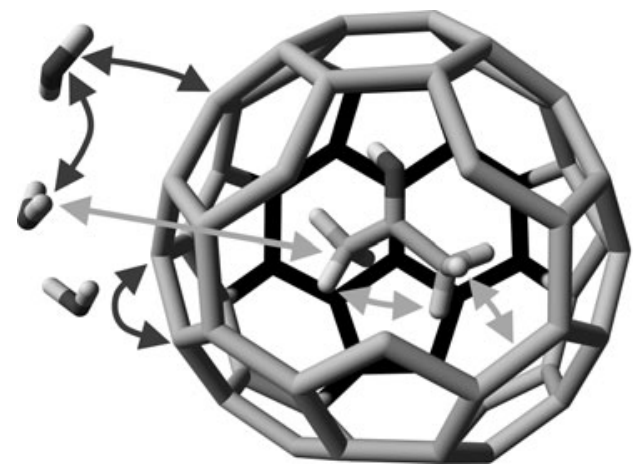

Fig. 1 Open view of 'extended' fullerene model. Light arrows depict ligand-surrounding energy terms, that is, ligand-solvent, ligand-ligand and ligand-protein interactions. Dark arrows depict surroundingsurrounding energy terms, that is, protein-protein, protein-solvent and the applied methodology should not only consider the (favourable) enthalpic interaction between the protein and the ligand and the (unfavourable) loss of configurational entropy, but also include the enthalpic and entropic contributions of (partial) desolvation [21].

Various computational methods to estimate $\Delta H$ and $\Delta S$ were assessed for reliability and efficiency. Apart from calculating the full enthalpy and entropy, we also investigated reduced terms by excluding the compensation in enthalpic and entropic contributions due to changes in the interactions within the surroundings of the ligand. From solvation studies, the reduced terms are known as the solute-solvent enthalpy and entropy [22, 23], which we here generalise to a ligand-surrounding enthalpy and entropy. Solvation studies have also shown that the exactly compensating solvent-solvent contributions may obscure a proper interpretation of enthalpic and entropic contribution to the free energy [24]. Also, in DD, the interpretation of the enthalpic and entropic contribution in terms of molecular interactions is often complex and possibly not unambiguous due to a cancellation of effects [25]. The convergence and use of the generalised reduced thermodynamic terms will be investigated and discussed. The methods will be outlined in the following theory section, followed by a description of the applied simulation methodology and settings and by a discussion of the results and the main conclusions.

\section{Theory}

The free enthalpy, $\Delta G$, enthalpy, $\Delta H$, and entropy, $\Delta S$ are connected via the Gibbs equation,

$\Delta G=\Delta H-T \Delta S$

where $T$ is the absolute temperature in Kelvin. For experiments and simulations at constant volume rather

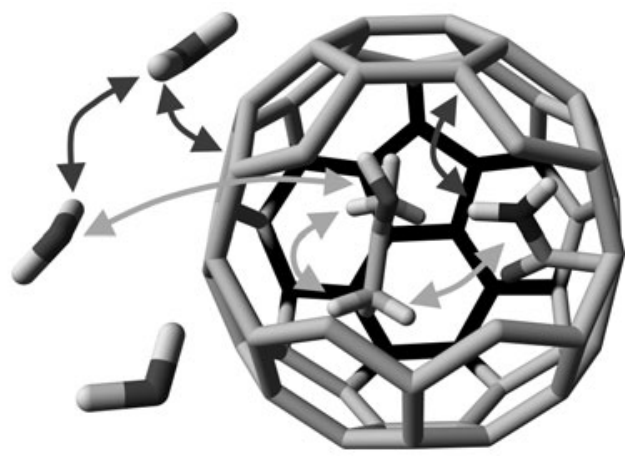

solvent-solvent interactions. The $\mathrm{C}_{\mathrm{APO}}$ model (left) emulates a mostly rigid hydrophobic protein pocket. The $\mathrm{C}_{\mathrm{HB}}$ model (right) has an additional $-(\mathrm{C}=\mathrm{O}) \mathrm{NH}_{2}$-group and emulates a mostly rigid hydrophobic protein pocket with hydrogen bond forming capability 
than constant pressure, we use the Helmholtz free energy $(\Delta A)$ and total energy $(\Delta E)$ to write,

$\Delta A=\Delta E-T \Delta S$

For ease of notation, and in line with the simulations performed in this work, we will restrict ourselves to the Helmholtz free energy below.

In the first approach, long molecular dynamics (MD) simulations at the end-states of a given process, for example, acetone or methanol in $\mathrm{C}_{\mathrm{APO}}$ or $\mathrm{C}_{\mathrm{HB}}$, were used to estimate $\Delta E$, while thermodynamic integration (TI) [26] was used to obtain $\Delta A$ using,

$\Delta A=\int_{0}^{1}\left\langle\frac{\partial \mathcal{H}(\lambda)}{\partial \lambda}\right\rangle_{\lambda} \mathrm{d} \lambda$,

where $\mathcal{H}$ is the Hamiltonian of the system and $\lambda$ is a coupling parameter that connects the initial state $(\lambda=0)$, the final state $(\lambda=1)$ and a series of intermediate states $(0<\lambda<1)$. The angular brackets represent an ensemble average obtained from a simulation at a state corresponding to the indicated $\lambda$-value. The integral of the (ensemble) average of the derivative of the Hamiltonian with respect to $\lambda$ gives $\Delta A . \Delta S$ is subsequently calculated from the estimated $\Delta A$ and $\Delta E$, using Eq. (2).

The second approach uses a different thermodynamic property which follows from Eq. (2):

$\Delta S=-\frac{\mathrm{d} \Delta A}{\mathrm{~d} T}$

Equation (4) implies that $\Delta S$ may be obtained from a linear regression over multiple $\Delta A$ estimates at different temperatures, obtained using, for example, TI.

The third approach estimates $\Delta S$ directly from TI [8], using Eq. (5):

$$
\begin{aligned}
\Delta S & =\frac{1}{k_{\mathrm{B}} \cdot T^{2}} \int_{0}^{1}\left\{\left\langle\frac{\partial \mathcal{H}(\lambda)}{\partial \lambda}\right\rangle_{\lambda}\langle\mathcal{H}(\lambda)\rangle_{\lambda}\right. \\
& \left.-\left\langle\frac{\partial \mathcal{H}(\lambda)}{\partial \lambda} \mathcal{H}(\lambda)\right\rangle_{\lambda}\right\} \cdot \mathrm{d} \lambda,
\end{aligned}
$$

where $k_{\mathrm{B}}$ is the Boltzmann constant. This equation is known to converge badly, because it involves correlations between the Hamiltonian and its derivative [8].

Next, we attempt to quantify the compensation of energetic and entropic contributions solely due to the surroundings of the ligand by defining reduced terms which stem from differences in interactions involving the ligand in two systems or states [22]. In solvation, such reduced terms were shown to converge more readily than the full energy and entropy differences [23]. We can generalise the approach by splitting the Hamiltonian $(\mathcal{H})$ into a $\lambda$ dependent term for the ligand-surrounding interaction $\left(\mathcal{H}_{\mathrm{ls}}\right)$ and a $\lambda$-independent term for the surrounding-surrounding energies $\left(\mathcal{H}_{\mathrm{ss}}\right)$,

$\mathcal{H}(\lambda)=\mathcal{H}_{\mathrm{ls}}(\lambda)+\mathcal{H}_{\mathrm{ss}}$

where $\mathcal{H}_{\mathrm{ls}}$ is defined as the sum of all non-bonded and bonded energy terms specific to interactions between the ligand and its surrounding. The non-bonded energy terms include ligand-ligand, ligand-protein and ligand-solvent Van der Waals and electrostatic interaction energy terms. The bonded energy terms include contributions from the ligands bonds, angles, improper dihedrals and dihedrals. $\mathcal{H}_{\text {ss }}$ refers to the surrounding-surrounding energies, here, made up of the protein-protein, protein-solvent and solvent-solvent interaction energies. Accordingly, we can write the energy difference as

$$
\begin{aligned}
\Delta E & =\langle\mathcal{H}\rangle_{1}-\langle\mathcal{H}\rangle_{0} \\
& =\Delta E_{\mathrm{ls}}+\Delta E_{\mathrm{ss}}=\left\langle\mathcal{H}_{\mathrm{ls}}\right\rangle_{1}-\left\langle\mathcal{H}_{\mathrm{ls}}\right\rangle_{0}+\left\langle\mathcal{H}_{\mathrm{ss}}\right\rangle_{1}-\left\langle\mathcal{H}_{\mathrm{ss}}\right\rangle_{0}
\end{aligned}
$$

Rewriting Eq. (5) while taking into account Eq. (6) now gives for the entropy difference

$$
\begin{aligned}
\Delta S & =\frac{1}{k_{\mathrm{B}} \cdot T^{2}} \int_{0}^{1}\left\{\left\langle\frac{\partial \mathcal{H}_{\mathrm{ls}}(\lambda)}{\partial \lambda}\right\rangle_{\lambda}\left\langle\mathcal{H}_{\mathrm{ls}}(\lambda)\right\rangle_{\lambda}-\left\langle\frac{\partial \mathcal{H}_{\mathrm{ls}}(\lambda)}{\partial \lambda} \mathcal{H}_{\mathrm{ls}}(\lambda)\right\rangle_{\lambda}\right. \\
& \left.+\left\langle\frac{\partial \mathcal{H}_{\mathrm{ls}}(\lambda)}{\partial \lambda}\right\rangle_{\lambda}\left\langle\mathcal{H}_{\mathrm{ss}}\right\rangle_{\lambda}-\left\langle\frac{\partial \mathcal{H}_{\mathrm{ls}}(\lambda)}{\partial \lambda} \mathcal{H}_{\mathrm{ss}}\right\rangle_{\lambda}\right\} \cdot \mathrm{d} \lambda
\end{aligned}
$$

We can also write the $\lambda$-derivative of the ensemble average of $\mathcal{H}_{\mathrm{ss}}$ as

$$
\begin{aligned}
\frac{\mathrm{d}}{\mathrm{d} \lambda}\left\langle\mathcal{H}_{\mathrm{ss}}\right\rangle_{\lambda}= & \frac{\mathrm{d}}{\mathrm{d} \lambda} \frac{\int \mathcal{H}_{\mathrm{ss}} \mathrm{e}^{-\mathcal{H}(\lambda) / k_{\mathrm{B}} T} \mathrm{~d} \mathbf{p d} \mathbf{r}}{\iint \mathrm{e}^{-\mathcal{H}(\lambda) / k_{\mathrm{B}} T} \mathrm{~d} \mathbf{p d} \mathbf{r}} \\
= & \iint \mathcal{H} \mathrm{ss} \frac{\mathrm{d}}{\mathrm{d} \lambda} \frac{\mathrm{e}^{-\mathcal{H}(\lambda) / k_{\mathrm{B}} T}}{\int \mathrm{e}^{-\mathcal{H}(\lambda) / k_{\mathrm{B}} T} \mathrm{~d} \mathbf{p d} \mathbf{r}} \mathrm{d} \mathbf{p} \mathrm{d} \mathbf{r} \\
= & \iint \mathcal{H}_{\mathrm{ss}}\left[\frac{-1}{k_{\mathrm{B}} T} \frac{\partial \mathcal{H}(\lambda)}{\partial \lambda} \frac{\mathrm{e}^{-\mathcal{H}(\lambda) / k_{\mathrm{B}} T}}{\int \mathrm{e}^{-\mathcal{H}(\lambda) / k_{\mathrm{B}} T} \mathrm{~d} \mathbf{p d} \mathbf{r}}\right. \\
& \left.-\frac{\mathrm{e}^{-\mathcal{H}(\lambda) / k_{\mathrm{B}} T} \iint \frac{-1}{k_{\mathrm{B}} T} \frac{\partial \mathcal{H}(\lambda)}{\partial \lambda} \mathrm{e}^{-\mathcal{H}(\lambda) / k_{\mathrm{B}} T} \mathrm{~d} \mathbf{p d} \mathbf{r}}{\left(\iint \mathrm{e}^{-\mathcal{H}(\lambda) / k_{\mathrm{B}} T} \mathrm{~d} \mathbf{p d r}\right)^{2}}\right] \mathrm{d} \mathbf{p d} \mathbf{r} \\
= & \frac{-1}{k_{\mathrm{B}} T}\left[\left\langle\mathcal{H}_{\mathrm{ss}} \frac{\partial \mathcal{H}_{\mathrm{ls}}(\lambda)}{\partial \lambda}\right\rangle_{\lambda}-\left\langle\mathcal{H}_{\mathrm{ss}}\right\rangle_{\lambda}\left\langle\frac{\partial \mathcal{H}_{\mathrm{ls}}(\lambda)}{\partial \lambda}\right\rangle_{\lambda}\right]
\end{aligned}
$$

where we explicitly write the ensemble average as a normalised integral over all positions (r) and momenta (p). We can now rewrite Eq. (8) as 


$$
\begin{aligned}
\Delta S & =\frac{1}{k_{\mathrm{B}} T^{2}} \int_{0}^{1}\left\{\left\langle\frac{\partial \mathcal{H}_{\mathrm{ls}}(\lambda)}{\partial \lambda}\right\rangle_{\lambda}\left\langle\mathcal{H}_{\mathrm{ls}}(\lambda)\right\rangle_{\lambda}\right. \\
& \left.-\left\langle\frac{\partial \mathcal{H}_{\mathrm{ls}}(\lambda)}{\partial \lambda} \mathcal{H}_{\mathrm{ls}}(\lambda)\right\rangle_{\lambda}\right\} \cdot \mathrm{d} \lambda+\frac{\Delta\left\langle\mathcal{H}_{\mathrm{ss}}\right\rangle}{T}=\Delta S_{\mathrm{ls}}+\frac{\Delta\left\langle\mathcal{H}_{\mathrm{ss}}\right\rangle}{T},
\end{aligned}
$$

defining the ligand-surrounding entropy $\Delta S_{\mathrm{ls}}$ as

$$
\begin{aligned}
\Delta S_{\mathrm{ls}}= & \frac{1}{k_{\mathrm{B}} T^{2}} \int_{0}^{1}\left\{\left\langle\frac{\partial \mathcal{H}_{\mathrm{ls}}(\lambda)}{\partial \lambda}\right\rangle_{\lambda}\left\langle\mathcal{H}_{\mathrm{ls}}(\lambda)\right\rangle_{\lambda}\right. \\
& \left.-\left\langle\frac{\partial \mathcal{H}_{\mathrm{ls}}(\lambda)}{\partial \lambda} \mathcal{H}_{\mathrm{ls}}(\lambda)\right\rangle_{\lambda}\right\} \cdot \mathrm{d} \lambda
\end{aligned}
$$

Together with the ligand-surrounding energy differences, $\Delta E_{\mathrm{ls}}$, in Eq. (7), we can write,

$\Delta A=\Delta E-T \Delta S=\Delta E_{\mathrm{ls}}-T \Delta S_{\mathrm{ls}}$

From Eq. (12), we can see that the free energy is only defined by the $\lambda$-dependent energy and the $\lambda$-dependent entropy while the energetic and entropic contributions from the $\lambda$-independent part, $\mathcal{H}_{\mathrm{ss}}$, exactly cancel in the free energy.

As will be outlined below, harmonic distance restraints were applied to restrain non-interacting dummy particles to a given position during the simulations. The contribution of these distance restraints to the free energy $\left(\Delta A_{\mathrm{r}}\right)$ and entropy $\left(\Delta S_{\mathrm{r}}\right)$ was calculated using Eqs. (13) and (14):

$$
\begin{aligned}
& \Delta A_{\mathrm{r}}=-k_{\mathrm{B}} T \cdot \ln \frac{V}{\left(\frac{2 \pi k_{\mathrm{B}} T}{K_{\mathrm{b}}}\right)^{\frac{3}{2}}} \\
& \Delta S_{\mathrm{r}}=-k_{\mathrm{B}} \cdot \ln \frac{V}{\left(\frac{2 \pi k_{\mathrm{B}} T}{K_{\mathrm{b}}}\right)^{\frac{3}{2}}}-\frac{3}{2} k_{\mathrm{B}}
\end{aligned}
$$

where $K_{\mathrm{b}}$ is the force constant used during the simulation and $V$ is the simulation box volume. Both equations are derived from comparing the partition functions of a threedimensional harmonic oscillator with the partition function of a freely translating particle [27, 28].

\section{Methods}

\subsection{Thermodynamic cycles}

A direct assessment of $\Delta A, \Delta E$ and $T \Delta S$ for ligand-protein binding from simulations of the actual binding event is very demanding [29], if not impossible [9]. However, $\Delta A$, $\Delta E$ and $T \Delta S$ are state functions. Therefore, it is possible to estimate relative changes of terms utilising thermodynamic cycles. A total of nine thermodynamic cycles composed of fifteen TI legs, shown in Fig. 2, were devised to study $\Delta A$ at a defined temperature. This allows us to carefully assess the convergence of the calculations, by determining the total free energy change along closed cycles, which should be $0 \mathrm{~kJ} \mathrm{~mol}^{-1}$ by definition. Moreover, we can determine the absolute and relative binding free energies of the two compounds or two host model systems by the appropriate combination of free energy terms. For instance, in a first TI, the acetone inside $\mathrm{C}_{\mathrm{HB}}\left(\mathrm{H}: \mathrm{A}_{\mathrm{q}}\right)$ changes into methanol $\left(\mathrm{H}: \mathrm{M}_{\mathrm{q}}\right)$ which yields $\Delta A_{\mathrm{m}}\left(\mathrm{H}: \mathrm{L}_{\mathrm{q}}\right)$. Performing similar simulations for the ligand-in-solvent [yielding $\Delta A_{\mathrm{m}}\left(\mathrm{L}_{\mathrm{q}}\right)$ ] allows for the calculation of the relative binding free energies $\left(\Delta \Delta A_{\mathrm{b}}\right)$ as the difference in binding free energy of methanol $\left[\Delta A_{\mathrm{b}}\left(\mathrm{H}: \mathrm{M}_{\mathrm{q}}\right)\right]$ and acetone $\left[\Delta A_{\mathrm{b}}\left(\mathrm{H}: \mathrm{A}_{\mathrm{q}}\right)\right]$ to the host $\mathrm{C}_{\mathrm{HB}}$ :

$$
\begin{aligned}
\Delta \Delta A_{\mathrm{b}} & =\Delta A_{\mathrm{b}}\left(\mathrm{H}: \mathrm{M}_{\mathrm{q}}\right)-\Delta A_{\mathrm{b}}\left(\mathrm{H}: \mathrm{A}_{\mathrm{q}}\right) \\
& =\Delta A_{\mathrm{m}}\left(\mathrm{H}: \mathrm{L}_{\mathrm{q}}\right)-\Delta A_{\mathrm{m}}\left(\mathrm{L}_{\mathrm{q}}\right)
\end{aligned}
$$

Similarly, from the starting state of acetone-in- $\mathrm{C}_{\mathrm{HB}}$ $\left(\mathrm{H}: \mathrm{A}_{\mathrm{q}}\right)$, charges may be removed from the acetone molecule (leading to $\mathrm{H}: \mathrm{A}_{\mathrm{n}}$ ), followed by removal of the Van der Waals interaction (leading to $\mathrm{H}: \mathrm{A}_{\mathrm{dr}}$ ). The resulting molecule, a so-called 'dummy' molecule, does not interact with its environment anymore, but still has a mass and a distance restraint which is introduced during the process to prevent the non-interacting acetone molecule from drifting through the complete simulation box, requiring extremely long simulations in the final stages of this TI leg [30, 31]. Equation (13) is used to calculate the contribution $\left[\Delta A_{\mathrm{r}}\left(\mathrm{H}: \mathrm{A}_{\mathrm{dr}}\right)\right]$ of the distance restraint to reach the state $\mathrm{H}: \mathrm{A}_{\mathrm{d}}$. The transfer of the non-interacting dummy molecule between solvent and the host system is not associated with a free energy change, that is, $\Delta A_{\mathrm{b}}\left(\mathrm{H}: \mathrm{A}_{\mathrm{d}}\right) \equiv 0$. Repeating these calculations for methanol-in- $\mathrm{C}_{\mathrm{HB}}$, for the ligands-in- $\mathrm{C}_{\mathrm{APO}}$ and the ligands-insolvent now permits estimation of the absolute binding free energy $\left(\Delta A_{\mathrm{b}}\right)$ of the free ligand to $\mathrm{C}_{\mathrm{APO}}$ or $\mathrm{C}_{\mathrm{HB}}$, e.g.:

$$
\begin{aligned}
\Delta A_{\mathrm{b}}\left(\mathrm{H}: \mathrm{A}_{\mathrm{q}}\right)= & \Delta A_{\mathrm{el}}\left(\mathrm{A}_{\mathrm{q}}\right)+\Delta A_{\mathrm{vdw}}\left(\mathrm{A}_{\mathrm{n}}\right)+\Delta A_{\mathrm{b}}\left(\mathrm{H}: \mathrm{A}_{\mathrm{d}}\right) \\
& -\Delta A_{\mathrm{r}}\left(\mathrm{H}: \mathrm{A}_{\mathrm{dr}}\right)-\Delta A_{\mathrm{vdw}}\left(\mathrm{H}: \mathrm{A}_{\mathrm{n}}\right) \\
& -\Delta A_{\mathrm{el}}\left(\mathrm{H}: \mathrm{A}_{\mathrm{q}}\right)
\end{aligned}
$$

Note that the term absolute binding free energy, commonly used in the field, still refers to a free energy difference along the binding process $[30,32]$.

Thermodynamic cycles can be used to determine internal consistency independent from experimental data. Many more cycles may be derived from Fig. 2, and a successful $\Delta A$ cycle closure is required before proceeding to calculate other terms.

A similar approach was used to study cycle closure for $\Delta S$ and $\Delta S_{1 \mathrm{~s}}$ where Eq. (14) was used instead of Eq. (13) for calculating the distance restraint contribution. 


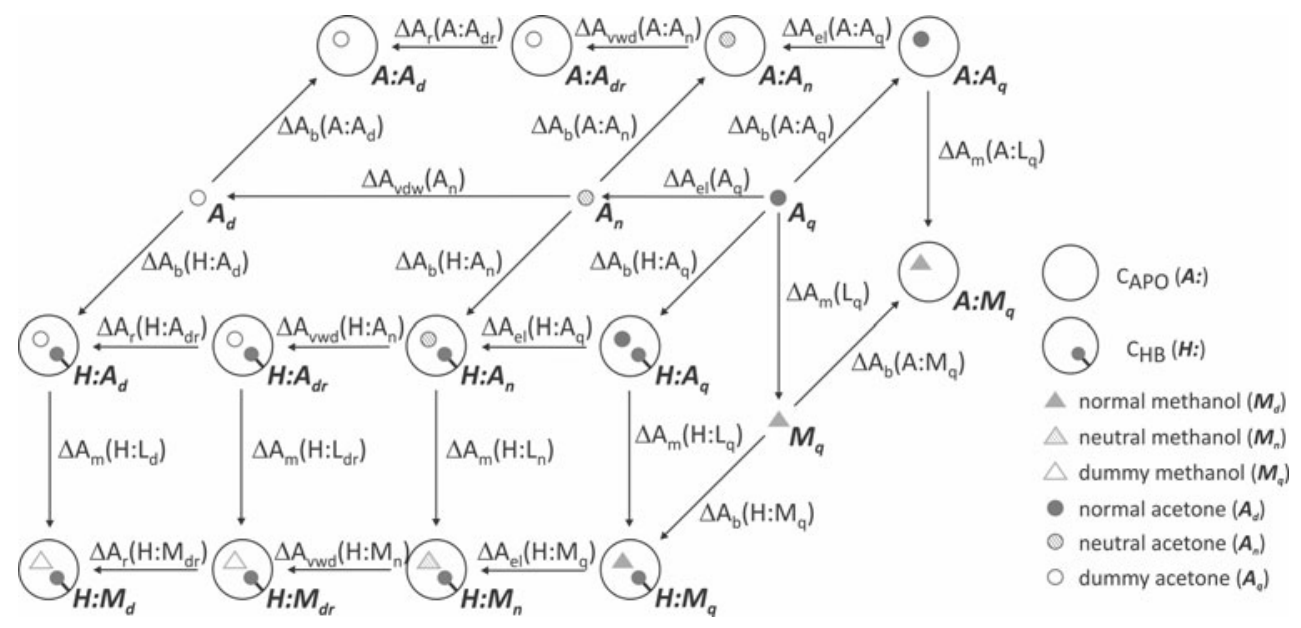

Fig. 2 A 3-dimensional representation of the free energy $(\Delta A)$ thermodynamic cycles. All arrows originate from a system in a certain state. With the exception of distance restraint terms $\left(\Delta A_{r}\right)$, all terms along the vertical and horizontal arrows were estimated using thermodynamic integration. Terms along diagonal arrows were calculated either by exploiting a thermodynamic cycle or by theoretical means. A ligand (L) can change into another ligand $\left(\Delta A_{\mathrm{m}}\right)$ or into its dummy state by the removal of electrostatic

\subsection{Simulation setup}

A single topology representation of both ligands (Fig. 3) was placed inside $\mathrm{C}_{\mathrm{APO}}$ and $\mathrm{C}_{\mathrm{HB}}$ and solvated in a periodic cubic box containing 1781 simple point charge (SPC) water molecules [33]. A similar setup for ligand-in- $\mathrm{C}_{\mathrm{HB}}$ requires 1792 SPC water molecules. Ligand-in-solvent simulations contained 1170 SPC water molecules. No counter ions were added. The GROMOS11 package for biomolecular simulations [34] was used for all simulations. Force field parameters were taken from the 54A7 unitedatom force field [35]. Hard-coded SPC water parameters were used to speed up the simulations. The number of particles, the volume and the temperature were kept constant during all simulations. Solvent and solute degrees of freedom were coupled separately to two temperature baths with a relaxation time of $0.1 \mathrm{ps}$ using the weak-coupling method [36]. We are aware of the fact that the weak-coupling does not result in energy fluctuations exactly corresponding to the canonical ensemble [37]. Therefore, the application of Eq. (5) may not lead to the exact entropy of the canonical ensemble. However, the aim of this study is not to establish the entropy for a (unphysical) host-guest model system, but to establish the convergence behaviour of Eq. (5). The mutation of acetone to methanol has been repeated using a Nosé-Hoover chains thermostat, leading to very comparable convergence behaviour (see Fig. S1 in supplementary material). The leap-frog algorithm [38] with a timestep of 2 fs was used. All bonds were constrained to their minimum energy values using the SHAKE algorithm [39]. Centre of mass translation was removed every 1,000 interactions $\left(\Delta A_{\mathrm{el}}\right)$, follow by the removal of Van der Waals interactions $\left(\Delta A_{\mathrm{vdw}}\right)$ while applying a distance restraint $\left(\Delta A_{\mathrm{r}}\right)$ that confines the dummy ligand to the geometrical centre of the 'extended' fullerene. The system and state from which values of a term are derived is always denoted between brackets. The ligand-in- $\mathrm{C}_{\mathrm{APO}}$ and ligand-in-solvent thermodynamic cycles are similar to the ligand-in$\mathrm{C}_{\mathrm{HB}}$ cycles, but are only partially illustrated

steps. All solute molecules were defined as separate energy groups and all solvent molecules defined as one energy group. Energies and free energy derivatives were written out every 50 steps in ligand-to-dummy simulations and every 100 steps in acetone to methanol simulations. In all TI simulations, non-bonded interactions involving ligand atoms are described by a Lennard-Jones soft-core parameter of 0.5 and a Coulomb-reaction-field soft-core parameter of $0.5 \mathrm{~nm}^{2}$ [40].

Non-bonded interactions were calculated using a triplerange cut-off scheme. Interactions up to a short-range distance of $0.8 \mathrm{~nm}$ were calculated at every timestep from a pairlist that was updated every 5 steps. At pairlist construction [41], interactions up to an intermediate range of $1.4 \mathrm{~nm}$ were also calculated and kept constant between updates. A reaction field contribution [42] was added to the forces and energies to account for a dielectric continuum with relative permittivity of 61 beyond the cut-off sphere of $1.4 \mathrm{~nm}[43]$.

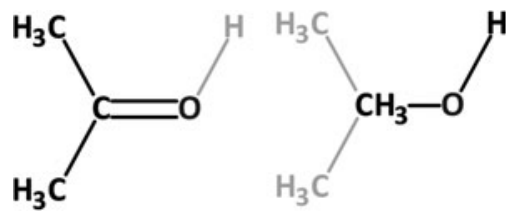

Fig. 3 Single topology representations of acetone (left) and methanol (right). Atoms in grey are non-interacting dummy particles. By switching the interaction function parameters, one molecule may be modified into the other or be switched off completely in a thermodynamic integration simulation 
Velocities corresponding to an initial temperature of $60 \mathrm{~K}$ were randomly assigned to all atoms before the equilibration process of each simulation, during which systems were heated to the desired temperature through gradual increase of temperature $(\Delta T=60 \mathrm{~K})$ while simultaneously decreasing an imposed position restraint on all solute atoms from $2.5 \times 10^{4}$ to $0 \mathrm{~kJ} \mathrm{~mol}^{-1} \mathrm{~nm}^{-2}$ in 5 discrete simulation steps of 20 ps each.

MD simulations of $100 \mathrm{~ns}$ were used to estimate $\Delta E$ and $\Delta E_{\mathrm{ls}}$ between the states acetone-in-solvent $\left(\mathrm{A}_{\mathrm{q}}\right)$, methanolin-solvent $\left(\mathrm{M}_{\mathrm{q}}\right), \mathrm{C}_{\mathrm{APO}}$-in-solvent $(\mathrm{A}), \mathrm{C}_{\mathrm{HB}}$-in-solvent $(\mathrm{H})$, acetone-in- $\mathrm{C}_{\mathrm{APO}}\left(\mathrm{A}: \mathrm{A}_{\mathrm{q}}\right)$, methanol-in- $\mathrm{C}_{\mathrm{APO}}\left(\mathrm{A}: \mathrm{M}_{\mathrm{q}}\right)$, acetone-in- $\mathrm{C}_{\mathrm{HB}} \quad\left(\mathrm{H}: \mathrm{A}_{\mathrm{q}}\right)$ and methanol-in- $\mathrm{C}_{\mathrm{HB}}\left(\mathrm{H}: \mathrm{M}_{\mathrm{q}}\right)$ at $300 \mathrm{~K}$.

TI simulations were performed by adjusting the force field parameters provided in the supplementary material between $\lambda=0$ and $\lambda=1$ for the corresponding states and monitoring the value of $\partial \mathcal{H} / \partial \lambda$ according to the GROMOS functional form [44]. Note that in GROMOS, 1,2- and 1,3neighbours are excluded from the non-bonded interactions and that the polar hydrogen atom does not have Van der Waals parameters. As the methyl groups in acetone have zero partial charges, the intramolecular non-bonded interactions amount to zero at all times. TI simulations were performed for the mutations $\Delta A_{\mathrm{m}}\left(\mathrm{L}_{\mathrm{q}}\right), \Delta A_{\mathrm{m}}\left(\mathrm{A}: \mathrm{L}_{\mathrm{q}}\right)$ and $\Delta A_{\mathrm{m}}\left(\mathrm{H}: \mathrm{L}_{\mathrm{q}}\right)$ in Fig. 2 using 51 equidistant $\lambda$-values. For the processes $\Delta A_{\mathrm{VdW}}$ and $\Delta A_{\mathrm{el}}$, the $\lambda$-value was increased by 0.04 between $\lambda=0$ and $\lambda=0.4$ and by 0.02 between $\lambda=0.4$ and $\lambda=1$, yielding 41 separate simulations. Preliminary calculations showed that convergence was sufficient in these simulations even though a slightly coarser approach was used (data not shown). $\Delta A_{\mathrm{m}}\left(\mathrm{L}_{\mathrm{q}}\right), \Delta A_{\mathrm{m}}\left(\mathrm{A}: \mathrm{L}_{\mathrm{q}}\right)$ and $\Delta A_{\mathrm{m}}\left(\mathrm{H}: \mathrm{L}_{\mathrm{q}}\right)$ were calculated at 220, 250, 280, 290, 300, $310,320,350$ and $380 \mathrm{~K}$ while $\Delta A_{\mathrm{VdW}}$ and $\Delta A_{\mathrm{el}}$ were only calculated at 280,300 and $320 \mathrm{~K}$. The simulations were performed for $10 \mathrm{~ns}$ at every $\lambda$-value at $300 \mathrm{~K}$ and $1.2 \mathrm{~ns}$ per $\lambda$-value at all other temperatures.

\subsection{Accuracy and efficiency determination}

The simulations described so far allow us to analyse the accuracy and precision of the various properties and approaches as a function of simulation time retrospectively. The total amount of simulation time was restricted to 100 ns while maintaining the most precise $\Delta A$ estimate in a non-automated manner. First, all $\lambda$-values that are believed to have minimal effect on the $\Delta A$ estimate were excluded. This was done by multiple iterations of plotting data, excluding $\lambda$-values at what seem to be linear regions and evaluating the influence of the excluded $\lambda$-values on $\Delta A$. This is followed by the determination of the minimal simulation time required at each remaining $\lambda$-value in two rounds which was achieved by monitoring $\partial \mathcal{H} / \partial \lambda$ as a function of time followed by a careful consideration of the trade-off between accuracy and simulation time. This way, the total simulation time for each calculated value was initially reduced to $100 \mathrm{~ns}$. The total simulation time was further reduced to $10 \mathrm{~ns}$ in a second round by reducing the lengths of the simulations by a factor 10 .

A similar approach was applied to optimise the calculation of $\Delta S_{1 \mathrm{~s}}$ with a given amount of overall simulation time. As $\Delta S$ is known to converge worse than $\Delta S_{\mathrm{ls}}$, the $\lambda$-values found to be optimal for $\Delta S_{\text {ls }}$ were also used for $\Delta S$. Data reduction for $\Delta E$ and $\Delta E_{\mathrm{ls}}$ was done by determining the minimal simulation time required per simulation.

Error estimates for the averages obtained from simulations were determined from block averaging and extrapolation to infinite block length [45]. Error estimates in the thermodynamic terms are subsequently obtained from standard propagation of the error estimates on the simulation averages [46].

\section{Results}

It is well-known that $\Delta A$ and $\Delta S$ converge differently [8]. Figure 4 shows the profiles of $\mathrm{dA} / \mathrm{d} \lambda, \mathrm{dS} / \mathrm{d} \lambda$ and $\mathrm{dS}_{1 \mathrm{~s}} / \mathrm{d} \lambda$ for the acetone to methanol mutation in solvent (see Fig. S2 and $\mathrm{S} 3$ in supplementary material for the profiles of $\mathrm{dA} / \mathrm{d} \lambda$, $\mathrm{dS} / \mathrm{d} \lambda$ and $\mathrm{dS}_{1 \mathrm{~s}} / \mathrm{d} \lambda$ in the host systems). As a minimal requirement for internal consistency, the thermodynamic cycle closure for $\Delta A$ was evaluated first. For the various thermodynamic cycles in Table 1, a cycle closure of maximally $2.5 \mathrm{~kJ} \mathrm{~mol}^{-1}\left(k_{\mathrm{B}} T\right)$ at $300 \mathrm{~K}$ was obtained. This observation also holds for the cycles studied at $320 \mathrm{~K}$. However, lowering the temperature to $280 \mathrm{~K}$ noticeably affects cycle closures, with deviations up to $4.5 \mathrm{~kJ} \mathrm{~mol}^{-1}$. Careful consideration of many possible factors that might affect the simulations at all three temperatures, including geometrical aspects of the ligand and its environment, various contributing energy terms and possible calculation errors, has led to the conclusion that the cycles are internally consistent. We will subsequently attempt to calculate $T \Delta S$ and $T \Delta S_{1 \mathrm{~s}}$ from the same simulation data at $300 \mathrm{~K}$. The cycles that do not close at $280 \mathrm{~K}$ most likely imply that due to reduced dynamics, additional sampling is still required for these systems at $280 \mathrm{~K}$.

The situation is different for the cycles at $300 \mathrm{~K}$ for $T \Delta S$, as obtained using Eq. (5), which are also presented in Table 1. Although some cycles seem close to closing, the error estimates clearly show that these values are far from converged. The main issue here is that the estimated errors are several orders of magnitude larger than the estimated value itself. In sharp contrast, the $T \Delta S_{1 \mathrm{~s}}$ cycles calculated using Eq. (11) are comparable to the $\Delta A$ cycles. The 


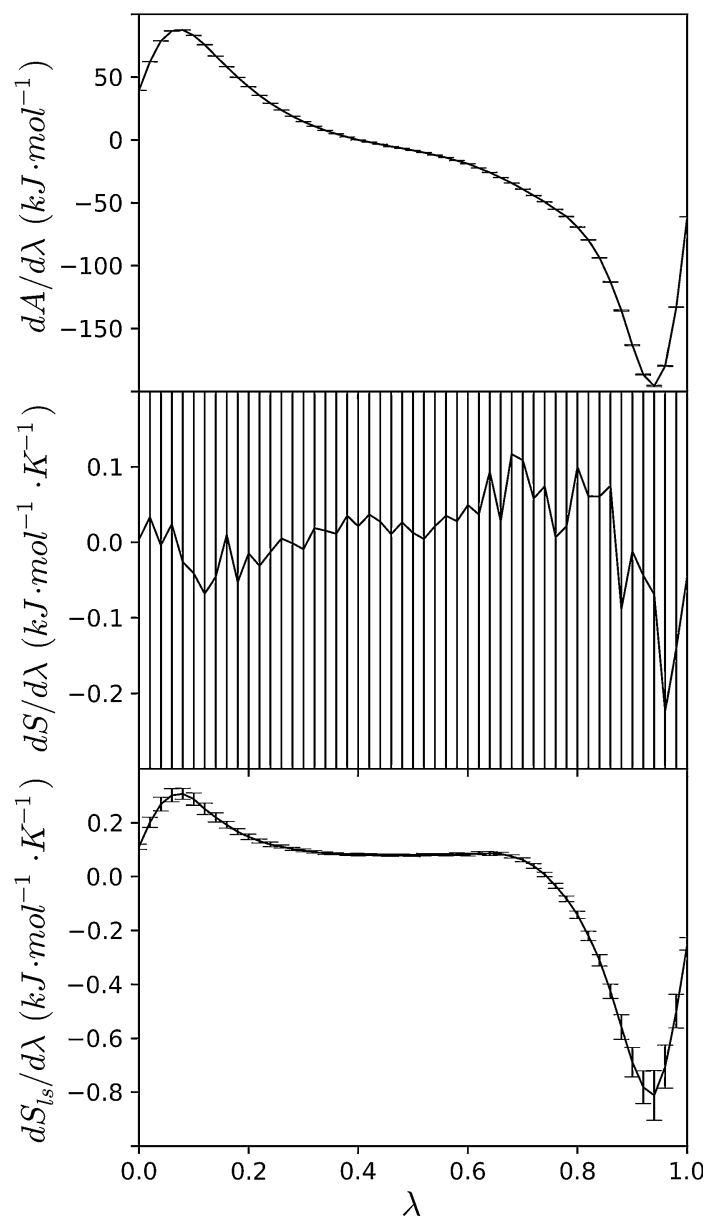

Fig. 4 The profiles of $\mathrm{dA} / \mathrm{d} \lambda, \mathrm{dS} / \mathrm{d} \lambda$ and $\mathrm{d} \mathrm{S}_{\mathrm{Is}} / \mathrm{d} \lambda$ for the acetone to methanol mutation in solvent. The profile of $\mathrm{dS} / \mathrm{d} \lambda$ is a clear example of a profile of a term that does not converge properly, note that the error bars fall off the scale of the graph, while the other two profiles, with a smoother curve, belong to terms that do converge to an acceptable degree

statistical errors are still about 10-fold larger than the estimated values, but are substantially smaller when compared to the estimated errors from the $T \Delta S$ cycles.

Table 2 presents estimates of $\Delta A, \Delta E, T \Delta S$ and $T \Delta S_{\mathrm{ls}}$ from all three different approaches for each system. We denote the direct application of Eq. (2) as approach I, the utilisation of multiple simulations at different temperature (Eq. 4) as approach II and the application of the thermodynamic integration formula (Eq. 5) as approach III. If all available simulation data are taken into consideration, approaches I and II (Eqs. 2, 4) seem to yield a similar $T \Delta S$ for ligand-in-solvent and ligand-in- $\mathrm{C}_{\mathrm{HB}}$, even though the estimated errors of the second approach are relatively large. Approach III is completely off, which was already observed for the thermodynamic cycle closures and confirms that $T \Delta S$ estimation using Eq. (5) remains a challenge, even with $510 \mathrm{~ns}$ of total simulation time for a very simple process as the mutation in water or in a purely hydrophobic environment.

Although $\Delta A$ estimation using TI is rather precise, using approach II to estimate $T \Delta S$ does not yield the most precise values. As can be seen from the curves in Fig. 5, the slopes of the Van 't Hoff plots are almost identical, independent of the simulation time invested. This indicates that the estimates of $T \Delta S$ using this approach is robust and only slightly affected by data reduction. The relatively large error estimates are due to the error propagation over the linear regression. The large overall amount of simulation time is divided over many individual simulations with (reasonable) error estimates, which are mostly additive in the final error estimates. In the other approaches, the overall simulation time is divided over fewer, longer simulations, more efficiently reducing the error estimates.

For the current system, approach I seems to yield the most precise estimates of the entropy. However, we have to note that this may be different for more realistic systems, for example, for a large flexible host molecule, undergoing slow conformational motion $\Delta E$ may not converge to a sufficient level to apply this approach. Approach II was previously applied efficiently for systems involving a smaller alchemical modification [47, 48].

The lower half of Table 2 presents the results for the reduced terms $\Delta E_{\mathrm{ls}}$ and $T \Delta S_{\mathrm{ls}}$. It can be seen that both $\Delta E_{\mathrm{ls}}$ and $T \Delta S_{\mathrm{ls}}$ converge substantially better than their full counterparts, $\Delta E$ and $\Delta S$. The ligand-in-solvent simulation data in Fig. 6 shows that both reduced terms require about 5 -fold less simulation time per $\lambda$-value to reach convergence and estimated errors for each term are substantially smaller. Ligand-in- $\mathrm{C}_{\mathrm{APO}}$ and ligand-in- $\mathrm{C}_{\mathrm{HB}}$ data (see Fig. S4 and S5 in supplementary material) follow a similar trend. The reduced noise for the ligand-surrounding energy and entropy indicates that the noise in the full energy and entropy estimates are mostly due to the surrounding-surrounding energy and entropy terms, which cancel exactly in the free energy, which hence converges more readily as well.

The values of $T \Delta S_{\text {ls }}$ as calculated from approach III (Eq. 11) are consistently $2.4-4.2 \mathrm{~kJ} \mathrm{~mol}^{-1}$ lower than the values calculated from approach II (Eq. 12). The discrepancy could be traced to the use of bond-length constraints in the simulation, that is, SHAKE, and a change of the $\mathrm{C}=\mathrm{O}$ bond of $0.123 \mathrm{~nm}$ in acetone to a $\mathrm{C}-\mathrm{O}$ bond of $0.153 \mathrm{~nm}$ in methanol. This leads to a slight change of the constraint forces as calculated in the SHAKE algorithm, which is included in the overall estimate of $\Delta A$ through the appropriate contribution to $\mathrm{dA} / \mathrm{d} \lambda$ [49]. As, however, a constraint to a (modified) minimum energy value is not reflected in an energy change, it will occur neither in the estimate of $\Delta E$ nor in the estimate of $T \Delta S$ using Eq. (5). The same holds for the calculations of the reduced terms $\Delta E_{\mathrm{ls}}$ and $T \Delta S_{\mathrm{ls}}$ using Eq. (11). Indeed, the free energy difference 
Table 1 Thermodynamic cycle closures for $\Delta A, T \Delta S$ and $T \Delta S_{\mathrm{ls}}$ in $\mathrm{kJ} \mathrm{mol}^{-1}$ (see Fig. 2 for explanations of abbreviations; $\mathrm{x}$ is a placeholder for $\mathrm{q}, \mathrm{n}, \mathrm{dr}$ or $\mathrm{d}$ )

\begin{tabular}{llrlrc}
\hline Cycle & $\Delta A(280 \mathrm{~K})$ & $\Delta A(300 \mathrm{~K})$ & $\Delta A(320 \mathrm{~K})$ & $T \Delta S(300 \mathrm{~K}) \mathrm{Eq} .(5)$ & $T \Delta S_{\text {ls }}(300 \mathrm{~K}) \mathrm{Eq} .(11)$ \\
\hline $\mathrm{A}_{\mathrm{x}} \mathrm{M}_{\mathrm{x}}$ (neutral) $^{\mathrm{b}}$ & $\mathrm{X}^{\mathrm{a}}$ & $-0.4 \pm 0.4$ & $\mathrm{X}^{\mathrm{a}}$ & $2.7 \pm 10,063$ & $0.8 \pm 11.3$ \\
$\mathrm{~A}_{\mathrm{x}} \mathrm{M}_{\mathrm{x}}$ (dummy) $^{\mathrm{c}}$ & $1.9 \pm 0.9$ & $-1.5 \pm 0.8$ & $1.1 \pm 0.8$ & $-11.1 \pm 18,111$ & $2.1 \pm 11.7$ \\
$\mathrm{~A}: \mathrm{A}_{\mathrm{x}} \mathrm{M}_{\mathrm{x}}$ (neutral) $^{\mathrm{d}}$ & $\mathrm{X}^{\mathrm{a}}$ & $0.4 \pm 0.5$ & $\mathrm{X}^{\mathrm{a}}$ & $0.5 \pm 17,646$ & $0.4 \pm 10.8$ \\
$\mathrm{~A}: \mathrm{A}_{\mathrm{x}} \mathrm{M}_{\mathrm{x}}$ (dummy) $^{\mathrm{e}}$ & $2.5 \pm 0.7$ & $-2.6 \pm 0.7$ & $-2.5 \pm 0.9$ & $-4.6 \pm 22,732$ & $4.3 \pm 6.7$ \\
$\mathrm{H}: \mathrm{A}_{\mathrm{x}} \mathrm{M}_{\mathrm{x}}$ (neutral) $^{\mathrm{f}}$ & $\mathrm{X}^{\mathrm{a}}$ & $0.2 \pm 0.8$ & $\mathrm{X}^{\mathrm{a}}$ & $-9.6 \pm 27,358$ & $0.9 \pm 18.6$ \\
$\mathrm{H}: \mathrm{A}_{\mathrm{x}} \mathrm{M}_{\mathrm{x}}$ (dummy) $^{\mathrm{g}}$ & $-1.9 \pm 1.2$ & $-1.7 \pm 1.1$ & $-1.8 \pm 1.4$ & $-4.5 \pm 37,344$ & $3.9 \pm 16.4$ \\
$\mathrm{~A}_{\mathrm{x}} \mathrm{M}_{\mathrm{x}}-\mathrm{A}: \mathrm{A}_{\mathrm{x}} \mathrm{M}_{\mathrm{x}}$ (overall) $^{\mathrm{h}}$ & $4.5 \pm 1.2$ & $1.1 \pm 1.1$ & $1.3 \pm 1.2$ & $-6.5 \pm 29,064$ & $-2.2 \pm 13.4$ \\
$\mathrm{~A}_{\mathrm{x}} \mathrm{M}_{\mathrm{x}}-\mathrm{H}: \mathrm{A}_{\mathrm{x}} \mathrm{M}_{\mathrm{x}}$ (overall) $^{\mathrm{i}}$ & $3.8 \pm 1.5$ & $0.2 \pm 1.4$ & $0.7 \pm 1.6$ & $-6.6 \pm 41,504$ & $-1.8 \pm 20.2$ \\
\hline
\end{tabular}

${ }^{a}$ Value not computed

${ }^{b} \mathrm{~A}_{\mathrm{n}} \rightarrow \mathrm{A}_{\mathrm{q}} \rightarrow \mathrm{M}_{\mathrm{q}} \rightarrow \mathrm{M}_{\mathrm{n}} \rightarrow \mathrm{A}_{\mathrm{n}}$-cycle

${ }^{c} \mathrm{~A}_{\mathrm{d}} \rightarrow \mathrm{A}_{\mathrm{n}} \rightarrow \mathrm{A}_{\mathrm{q}} \rightarrow \mathrm{M}_{\mathrm{q}} \rightarrow \mathrm{M}_{\mathrm{n}} \rightarrow \mathrm{M}_{\mathrm{d}} \rightarrow \mathrm{A}_{\mathrm{d}}$-cycle

${ }^{\mathrm{d}} \mathrm{A}: \mathrm{A}_{\mathrm{n}} \rightarrow \mathrm{A}: \mathrm{A}_{\mathrm{q}} \rightarrow \mathrm{A}: \mathrm{M}_{\mathrm{q}} \rightarrow \mathrm{A}: \mathrm{M}_{\mathrm{n}} \rightarrow \mathrm{A}: \mathrm{A}_{\mathrm{n}}$-cycle

${ }^{\text {e }} \mathrm{A}: \mathrm{A}_{\mathrm{d}} \rightarrow \mathrm{A}: \mathrm{A}_{\mathrm{dr}} \rightarrow \mathrm{A}: \mathrm{A}_{\mathrm{n}} \rightarrow \mathrm{A}: \mathrm{A}_{\mathrm{q}} \rightarrow \mathrm{A}: \mathrm{M}_{\mathrm{q}} \rightarrow \mathrm{A}: \mathrm{M}_{\mathrm{n}} \rightarrow \mathrm{A}: \mathrm{M}_{\mathrm{dr}} \rightarrow \mathrm{A}: \mathrm{M}_{\mathrm{d}} \rightarrow \mathrm{A}: \mathrm{A}_{\mathrm{d}}-$ cycle

${ }^{\mathrm{f}} \mathrm{H}: \mathrm{A}_{\mathrm{n}} \rightarrow \mathrm{H}: \mathrm{A}_{\mathrm{q}} \rightarrow \mathrm{H}: \mathrm{M}_{\mathrm{q}} \rightarrow \mathrm{H}: \mathrm{M}_{\mathrm{n}} \rightarrow \mathrm{H}: \mathrm{A}_{\mathrm{n}}$-cycle

${ }^{\mathrm{g}} \mathrm{H}: \mathrm{A}_{\mathrm{d}} \rightarrow \mathrm{H}: \mathrm{A}_{\mathrm{dr}} \rightarrow \mathrm{H}: \mathrm{A}_{\mathrm{n}} \rightarrow \mathrm{H}: \mathrm{A}_{\mathrm{q}} \rightarrow \mathrm{H}: \mathrm{M}_{\mathrm{q}} \rightarrow \mathrm{H}: \mathrm{M}_{\mathrm{n}} \rightarrow \mathrm{H}: \mathrm{M}_{\mathrm{dr}} \rightarrow \mathrm{H}: \mathrm{M}_{\mathrm{d}} \rightarrow \mathrm{H}: \mathrm{A}_{\mathrm{d}}-$ cycle

${ }^{\mathrm{h}} \mathrm{A}: \mathrm{A}_{\mathrm{d}} \rightarrow \mathrm{A}: \mathrm{A}_{\mathrm{dr}} \rightarrow \mathrm{A}: \mathrm{A}_{\mathrm{n}} \rightarrow \mathrm{A}: \mathrm{A}_{\mathrm{q}} \rightarrow \mathrm{A}: \mathrm{M}_{\mathrm{q}} \rightarrow \mathrm{A}: \mathrm{M}_{\mathrm{n}} \rightarrow \mathrm{A}: \mathrm{M}_{\mathrm{dr}} \rightarrow \mathrm{A}: \mathrm{M}_{\mathrm{d}} \rightarrow \mathrm{M}_{\mathrm{d}} \rightarrow \mathrm{M}_{\mathrm{n}} \rightarrow \mathrm{M}_{\mathrm{q}} \rightarrow \mathrm{A}_{\mathrm{q}} \rightarrow \mathrm{A}_{\mathrm{n}} \rightarrow \mathrm{A}$ d $\rightarrow \mathrm{A}: \mathrm{A}_{\mathrm{d}}-\mathrm{cycle}$

${ }^{\mathrm{i}} \mathrm{H}: \mathrm{A}_{\mathrm{d}} \rightarrow \mathrm{H}: \mathrm{A}_{\mathrm{dr}} \rightarrow \mathrm{H}: \mathrm{A}_{\mathrm{n}} \rightarrow \mathrm{H}: \mathrm{A}_{\mathrm{q}} \rightarrow \mathrm{H}: \mathrm{M}_{\mathrm{q}} \rightarrow \mathrm{H}: \mathrm{M}_{\mathrm{n}} \rightarrow \mathrm{H}: \mathrm{M}_{\mathrm{dr}} \rightarrow \mathrm{H}: \mathrm{M}_{\mathrm{d}} \rightarrow \mathrm{M}_{\mathrm{d}} \rightarrow \mathrm{M}_{\mathrm{n}} \rightarrow \mathrm{M}_{\mathrm{q}} \rightarrow \mathrm{A}_{\mathrm{q}} \rightarrow \mathrm{A}_{\mathrm{n}} \rightarrow \mathrm{A}_{\mathrm{d}} \rightarrow \mathrm{H}: \mathrm{A}_{\mathrm{d}}-\mathrm{cycle}$

Table $2 \Delta A, \Delta E, T \Delta S$ and $T \Delta S_{\mathrm{ls}}$ in $\mathrm{kJ} \mathrm{mol}^{-1}$ from different approaches for the mutation of acetone to methanol

\begin{tabular}{llllll}
\hline & $\Delta A_{\mathrm{m}}$ & $\Delta E$ & $T \Delta S$ [approach I; Eq. (2)] & $T \Delta S$ [approach II; Eq. (4)] & $T \Delta S$ [approach III; Eq. (5)] \\
\hline Simulation time $^{\mathrm{d}}$ & $510 \mathrm{~ns}$ & $200 \mathrm{~ns}$ & $710 \mathrm{~ns}$ & $1,009.8 \mathrm{~ns}$ & $510 \mathrm{~ns}$ \\
$1: \mathrm{A}_{\mathrm{q}} \rightarrow \mathrm{M}_{\mathrm{q}}$ & $-19.5 \pm 0.2$ & $-6.3 \pm 0.6$ & $13.2 \pm 0.6$ & $14.0 \pm 11.2$ & $2.2 \pm 4,230$ \\
$2: \mathrm{A}: \mathrm{A}_{\mathrm{q}} \rightarrow \mathrm{A}: \mathrm{M}_{\mathrm{q}}$ & $11.7 \pm 0.1$ & $29.7 \pm 0.8$ & $18.0 \pm 0.8$ & $11.6 \pm 7.0$ & $1.3 \pm 4,444$ \\
3: $\mathrm{H}: \mathrm{A}_{\mathrm{q}} \rightarrow \mathrm{H}: \mathrm{M}_{\mathrm{q}}$ & $-15.1 \pm 0.2$ & $-4.5 \pm 0.8$ & $10.7 \pm 0.8$ & $-2.4 \pm 13.2$ & $2.1 \pm 7,857$ \\
Relative 2-1 & $31.2 \pm 0.2$ & $36.0 \pm 1.0$ & $4.8 \pm 1.0$ & $-1.8 \pm 16.2$ & $-1.0 \pm 6,135$ \\
Relative 3-1 & $4.4 \pm 0.3$ & $1.8 \pm 1.0$ & $-2.5 \pm 1.0$ & $-0.2 \pm 8,923$ \\
\hline & & $\Delta E_{\mathrm{ls}}$ & $T \Delta S_{\text {ls }}$ [approach I; Eq. (12)] & $T \Delta S_{\text {ls }}$ [approach III; Eq. (11)] \\
\hline Reduced terms & & & & $-4.5 \pm 5.03$ \\
4: $\mathrm{A}_{\mathrm{q}} \rightarrow \mathrm{M}_{\mathrm{q}}$ & $\mathrm{X}^{\mathrm{c}}$ & $-21.6 \pm 0.0$ & $-2.1 \pm 0.2$ & $\mathrm{X}^{\mathrm{b}}$ & $6.3 \pm 2.5$ \\
5: A: $\mathrm{A}_{\mathrm{q}} \rightarrow \mathrm{A}: \mathrm{M}_{\mathrm{q}}$ & $\mathrm{X}^{\mathrm{c}}$ & $22.2 \pm 0.1$ & $10.5 \pm 0.1$ & $\mathrm{X}^{\mathrm{b}}$ & $10.6 \pm 5.6$ \\
6: H: $\mathrm{A}_{\mathrm{q}} \rightarrow \mathrm{H}: \mathrm{M}_{\mathrm{q}}$ & $\mathrm{X}^{\mathrm{c}}$ & $-1.1 \pm 0.2$ & $14.1 \pm 0.1$ & $\mathrm{X}^{\mathrm{b}}$ & $10.8 \pm 5.6$ \\
Relative 5-4 & $\mathrm{X}^{\mathrm{b}}$ & $43.8 \pm 0.4$ & $12.6 \pm 0.2$ & $\mathrm{X}^{\mathrm{b}}$ & $15.1 \pm 7.5$ \\
Relative 6-4 & $\mathrm{X}^{\mathrm{b}}$ & $20.5 \pm 0.5$ & $16.1 \pm 0.1$ & $\mathrm{X}^{\mathrm{b}}$ & \\
\hline
\end{tabular}

a $\Delta \Delta A(\Delta \Delta E, T \Delta \Delta S)$ is calculated from two $\Delta A(\Delta E, T \Delta S)$ values of which one is from ligand-in-solvent, and the other is from either ligand-in$\mathrm{C}_{\mathrm{APO}}$ or ligand-in- $\mathrm{C}_{\mathrm{HB}}$, indicated by the numbers of the previous lines. This also applies to the reduced properties

b Value not computed

c No reduced term exists; full term is used

d The total amount of simulation time used to calculate the values in the corresponding column. Relative entropy terms require twice the amount of total simulation time

between rigid rotors of lengths $0.123 \mathrm{~nm}$ and $0.153 \mathrm{~nm}$ amount to about $1 \mathrm{~kJ} \mathrm{~mol}^{-1}$ [27]. From a calculation of $\Delta A_{\mathrm{m}}\left(\mathrm{A}_{\mathrm{q}}\right)$ without applying SHAKE on the solute, a value of $-21.8 \mathrm{~kJ} \mathrm{~mol}^{-1}$ is obtained $\left(-19.5 \mathrm{~kJ} \mathrm{~mol}^{-1}\right.$ with SHAKE), explaining the difference of $2.4 \mathrm{~kJ} \mathrm{~mol}^{-1}$ for the ligand-in-solvent state. Note, however, that the differences in $T \Delta S_{\text {ls }}$ largely cancel in the relative entropy changes $T \Delta \Delta S_{\text {ls }}$ (Table 2). This suggests that both approaches II and III (Eqs. 11, 12) are suitable to estimate $T \Delta \Delta S_{\text {ls }}$ consistently which in turn is interesting for computational DD. However, the full $T \Delta \Delta S$ term does not seem to be directly comparable to the reduced $T \Delta \Delta S_{\mathrm{ls}}$ term. 


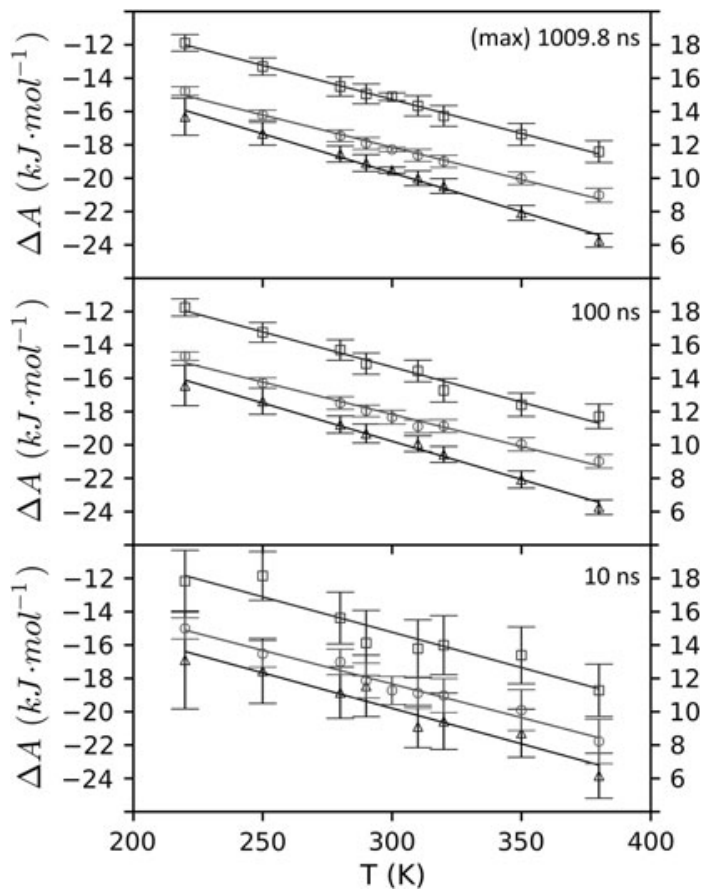

Fig. 5 Simulations were performed at 220, 250, 280, 290, 300, 310, 320,350 and $380 \mathrm{~K}$ for $\Delta A_{\mathrm{m}}\left(\mathrm{H}: \mathrm{L}_{\mathrm{q}}\right)$ (open square; left $y$-axis), $\Delta A_{\mathrm{m}}\left(\mathrm{A}: \mathrm{L}_{\mathrm{q}}\right)$ (open circle; right $y$-axis) and $\Delta A_{\mathrm{m}}\left(\mathrm{L}_{\mathrm{q}}\right)$ (open triangle; left $y$-axis). The maximum simulation time spent is $10 \mathrm{~ns}$ per $\lambda$-value at $300 \mathrm{~K}$ and $1.2 \mathrm{~ns}$ per $\lambda$-value at the other temperatures. A strong correlation is observed $\left(r^{2}=0.99\right)$ for all three systems at maximum simulation time with a slight deterioration when the summed up simulation time for all temperatures is reduced to $100 \mathrm{~ns}$ $\left(r^{2}=0.98-0.99\right)$. Differences between the systems $\mathrm{H}: \mathrm{L}_{\mathrm{q}}, \mathrm{A}: \mathrm{L}_{\mathrm{q}}$ and $\mathrm{L}_{\mathrm{q}}$ become noticeable upon further reduction of the net simulation time to $10 \mathrm{~ns}$ yielding $r^{2}=0.93, r^{2}=0.97$ and $r^{2}=0.90$, respectively

The setup for calculating the values in Table 2 for each approach is quite inefficient, and different amounts of simulation time were used in the various approaches, possibly obscuring a fair comparison of their efficiencies. Therefore, the question arises whether the same is achievable using $100 \mathrm{~ns}$ overall simulation time per calculated $T \Delta S$ or $T \Delta S_{1 \mathrm{~s}}$. This allows for a fair comparison of the precision to be reached by the various approaches. The results from a careful reduction of simulation data are presented in Table 3 and show that similar trends are still observed. The error estimates increase slightly and the values vary somewhat, but remain very similar with the exception of $\Delta E$ from ligand-in- $\mathrm{C}_{\mathrm{APO}}$. A reduction of the simulation time seems to affect the full terms more than the reduced terms, and a $100 \mathrm{~ns}$ overall simulation time still seems adequate. Do keep in mind that this is not automated and biases may have been introduced during the manual data reduction process.

Reducing the overall simulation time further to $10 \mathrm{~ns}$ per $T \Delta S$ or $T \Delta S_{\mathrm{ls}}$, results in the values presented in Table 4 . Again, the full terms seem most affected while the reduced

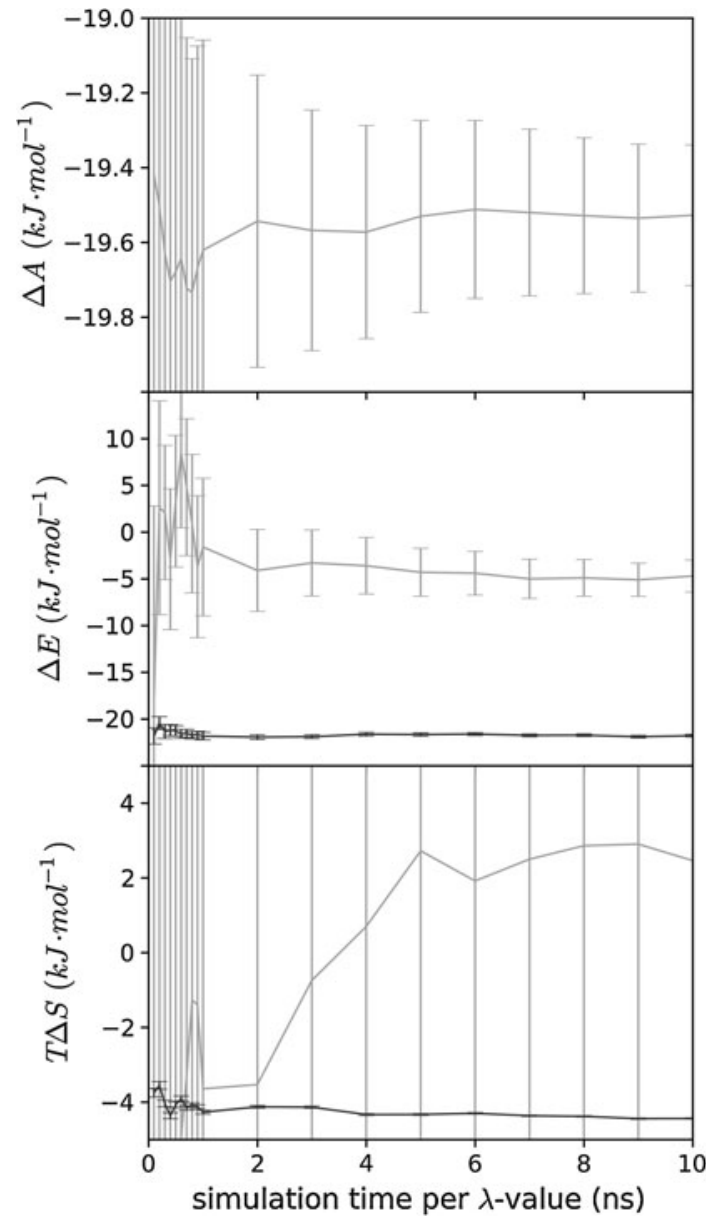

Fig. 6 Convergence of $\Delta A_{\mathrm{m}}, \Delta E$ and $T \Delta S$ as function of simulation time in the ligand-in-solvent simulation. $\Delta A_{\mathrm{m}}$ and $T \Delta S$ are calculated using Eqs. (3) and (5)/(11) over $51 \lambda$-values, respectively. $\Delta E$ is calculated using Eq. (7) over the simulations at $\lambda=1$ and $\lambda=0$. These plots with error bars at each value clearly show a substantially better convergence of the reduced terms $\Delta E_{\mathrm{ls}}$ and $T \Delta S_{\mathrm{ls}}$ (black) in comparison with the normal full terms $\Delta E$ and $T \Delta S$ (grey). Data were collected at every $100 \mathrm{ps}$ for the first ns and subsequently at every $1 \mathrm{~ns}$

terms are less susceptible. The best convergence seems to be obtained for $\Delta A$, closely followed by the reduced $\Delta E_{\mathrm{ls}}$ and $T \Delta S_{1 \mathrm{~s}}$ terms, while the full $\Delta E$ and $T \Delta S$ terms deviate more, due to insufficient sampling of the solvent-solvent degrees of freedom.

\section{Discussion}

The mutation of acetone to methanol was simulated in different surrounding environments: pure solvent, bound to $\mathrm{C}_{\mathrm{APO}}$ in water and bound to $\mathrm{C}_{\mathrm{HB}}$ in water. The cavity in the first host model represents a relatively large hydrophobic cavity, while the cavity in the second host model has a more hydrophilic character and is smaller in size. In the current force field (parameter set 54A7, see supplementary 
Table $3 \Delta A, \Delta E, T \Delta S$ and $T \Delta S_{\mathrm{ls}}$ in $\mathrm{kJ} \mathrm{mol}^{-1}$ calculated at $300 \mathrm{~K}$ using different methods when restricted to an overall simulation time of $100 \mathrm{~ns}$

\begin{tabular}{|c|c|c|c|c|c|}
\hline & $\Delta A_{\mathrm{m}}^{\mathrm{d}}$ & $\Delta E^{\mathrm{d}}$ & $T \Delta S$ [approach I; Eq. (2)] & $T \Delta S$ [approach II; Eq. (4)] & $T \Delta S$ [approach III; Eq. (5)] \\
\hline $1: \mathrm{A}_{\mathrm{q}} \rightarrow \mathrm{M}_{\mathrm{q}}$ & $-20.0 \pm 0.3$ & $-5.3 \pm 1.02$ & $14.7 \pm 1.2$ & $13.7 \pm 12.2$ & $7.8 \pm 4,418$ \\
\hline $2: \mathrm{A}: \mathrm{A}_{\mathrm{q}} \rightarrow \mathrm{A}: \mathrm{M}_{\mathrm{q}}$ & $11.6 \pm 0.2$ & $25.5 \pm 1.7$ & $13.9 \pm 1.7$ & $11.6 \pm 7.6$ & $2.7 \pm 4,562$ \\
\hline 3: $\mathrm{H}: \mathrm{A}_{\mathrm{q}} \rightarrow \mathrm{H}: \mathrm{M}_{\mathrm{q}}$ & $-15.2 \pm 0.4$ & $-3.4 \pm 1.5$ & $11.8 \pm 1.6$ & $12.6 \pm 12.7$ & $1.0 \pm 8,259$ \\
\hline Relative $2-1^{\mathrm{a}}$ & $31.6 \pm 0.4$ & $30.8 \pm 2.0$ & $-0.8 \pm 2.1$ & $-2.2 \pm 14.4$ & $-5.0 \pm 6,351$ \\
\hline \multirow[t]{2}{*}{ Relative $3-1^{a}$} & $4.8 \pm 0.5$ & $1.9 \pm 1.8$ & $-3.0 \pm 2.0$ & $-1.1 \pm 17.6$ & $-6.8 \pm 9,366$ \\
\hline & & $\Delta E_{\mathrm{ls}}$ & $T \Delta S_{\text {ls }}$ [approach I; Eq. (12)] & & $T \Delta S_{\text {ls }}$ [approach III; Eq. (11)] \\
\hline \multicolumn{6}{|l|}{ Reduced terms } \\
\hline 4: $\mathrm{A}_{\mathrm{q}} \rightarrow \mathrm{M}_{\mathrm{q}}$ & $\mathrm{X}^{\mathrm{c}}$ & $-21.6 \pm 0.1$ & $-1.6 \pm 0.3$ & $\mathrm{X}^{\mathrm{b}}$ & $-4.9 \pm 5.2$ \\
\hline 5: $\mathrm{A}: \mathrm{A}_{\mathrm{q}} \rightarrow \mathrm{A}: \mathrm{M}_{\mathrm{q}}$ & $X^{c}$ & $22.4 \pm 0.1$ & $10.8 \pm 0.2$ & $\mathrm{X}^{\mathrm{b}}$ & $6.1 \pm 2.5$ \\
\hline 6: $\mathrm{H}: \mathrm{A}_{\mathrm{q}} \rightarrow \mathrm{H}: \mathrm{M}_{\mathrm{q}}$ & $\mathrm{X}^{\mathrm{c}}$ & $-1.0 \pm 0.1$ & $14.2 \pm 0.4$ & $\mathrm{X}^{\mathrm{b}}$ & $11.5 \pm 6.0$ \\
\hline Relative $5-4^{\mathrm{a}}$ & $\mathrm{X}^{\mathrm{b}}$ & $44.0 \pm 0.1$ & $12.4 \pm 0.4$ & $\mathrm{X}^{\mathrm{b}}$ & $11.0 \pm 5.8$ \\
\hline Relative $6-4^{\mathrm{a}}$ & $X^{b}$ & $20.6 \pm 0.1$ & $15.8 \pm 0.5$ & $\mathrm{X}^{\mathrm{b}}$ & $16.4 \pm 8.0$ \\
\hline
\end{tabular}

${ }^{\text {a }} \Delta \Delta A(\Delta \Delta E, T \Delta \Delta S)$ is calculated from two $\Delta A(\Delta E, T \Delta S)$ values of which one is from ligand-in-solvent, and the other is from either ligand-in$\mathrm{C}_{\mathrm{APO}}$ or ligand-in- $\mathrm{C}_{\mathrm{HB}}$, indicated by the numbers of the previous lines. This also applies to the reduced properties

b Value not computed

c No reduced term exists; full term is used

d The amount of simulation time and choice of the $\lambda$-points contributing to the final $\Delta A$ or $\Delta E$ value is different per $T \Delta \Delta S$ and $T \Delta S$ term

Table $4 \Delta A, \Delta E, T \Delta S$ and $T \Delta S_{\mathrm{ls}}$ in $\mathrm{kJ} \mathrm{mol}^{-1}$ calculated at $300 \mathrm{~K}$ using different methods when restricted to an overall simulation time of $10 \mathrm{~ns}$

\begin{tabular}{|c|c|c|c|c|c|}
\hline & $\Delta A_{\mathrm{m}}^{\mathrm{d}}$ & $\Delta E^{\mathrm{d}}$ & $T \Delta S$ [approach I; Eq. (2)] & $T \Delta S$ [approach II; Eq. (4)] & $T \Delta S$ [approach III; Eq. (5)] \\
\hline 1: $\mathrm{A}_{\mathrm{q}} \rightarrow \mathrm{M}_{\mathrm{q}}$ & $-19.8 \pm 0.7$ & $-1.9 \pm 4.1$ & $17.9 \pm 4.1$ & $12.8 \pm 33.8$ & $3.3 \pm 13,495$ \\
\hline $2: \mathrm{A}: \mathrm{A}_{\mathrm{q}} \rightarrow \mathrm{A}: \mathrm{M}_{\mathrm{q}}$ & $11.5 \pm 0.5$ & $31.9 \pm 6.1$ & $20.3 \pm 6.1$ & $12.1 \pm 20.6$ & $12.7 \pm 14,619$ \\
\hline 3: $\mathrm{H}: \mathrm{A}_{\mathrm{q}} \rightarrow \mathrm{H}: \mathrm{M}_{\mathrm{q}}$ & $-15.0 \pm 0.9$ & $-10.2 \pm 4.2$ & $4.8 \pm 4.3$ & $12.7 \pm 32.8$ & $-3.1 \pm 22,608$ \\
\hline Relative $2-1^{a}$ & $31.3 \pm 0.9$ & $33.8 \pm 7.3$ & $2.5 \pm 7.4$ & $-0.7 \pm 39.5$ & $9.4 \pm 19,895$ \\
\hline \multirow[t]{2}{*}{ Relative $3-1^{\text {a }}$} & $4.8 \pm 1.1$ & $-8.3 \pm 5.9$ & $-13.1 \pm 6.0$ & $-0.1 \pm 47.1$ & $-6.4 \pm 26,329$ \\
\hline & & $\Delta E_{\mathrm{ls}}$ & $T \Delta S_{\text {ls }}$ [approach I; Eq. (12)] & & $T \Delta S_{\text {ls }}$ [approach III; Eq. (11) \\
\hline \multicolumn{6}{|l|}{ Reduced terms } \\
\hline 4: $\mathrm{A}_{\mathrm{q}} \rightarrow \mathrm{M}_{\mathrm{q}}$ & $\mathrm{X}^{\mathrm{c}}$ & $-22.0 \pm 0.2$ & $-3.2 \pm 0.8$ & $\mathrm{X}^{\mathrm{b}}$ & $-5.7 \pm 15.6$ \\
\hline $5: \mathrm{A}: \mathrm{A}_{\mathrm{q}} \rightarrow \mathrm{A}: \mathrm{M}_{\mathrm{q}}$ & $X^{c}$ & $22.5 \pm 0.4$ & $10.9 \pm 0.6$ & $\mathrm{X}^{\mathrm{b}}$ & $6.3 \pm 8.3$ \\
\hline 6: $\mathrm{H}: \mathrm{A}_{\mathrm{q}} \rightarrow \mathrm{H}: \mathrm{M}_{\mathrm{q}}$ & $X^{c}$ & $-1.0 \pm 0.2$ & $14.0 \pm 0.9$ & $\mathrm{X}^{\mathrm{b}}$ & $6.8 \pm 21.2$ \\
\hline Relative $5-4^{\mathrm{a}}$ & $\mathrm{X}^{\mathrm{b}}$ & $44.5 \pm 0.4$ & $14.1 \pm 1.0$ & $\mathrm{X}^{\mathrm{b}}$ & $12.0 \pm 17.7$ \\
\hline Relative $6-4^{\mathrm{a}}$ & $X^{b}$ & $21.0 \pm 0.3$ & $17.1 \pm 1.2$ & $\mathrm{X}^{\mathrm{b}}$ & $12.5 \pm 26.4$ \\
\hline
\end{tabular}

${ }^{\text {a }} \Delta \Delta A(\Delta \Delta E, T \Delta \Delta S)$ is calculated from two $\Delta A(\Delta E, T \Delta S)$ values of which one is from ligand-in-solvent, and the other is from either ligand-in$\mathrm{C}_{\mathrm{APO}}$ or ligand-in- $\mathrm{C}_{\mathrm{HB}}$, indicated by the numbers of the previous lines. This also applies to the reduced properties

b Value not computed

${ }^{c}$ No reduced term exists; full term is used

d The amount of simulation time and choice of the $\lambda$-points contributing to the final $\Delta A$ or $\Delta E$ value is different per $T \Delta \Delta S$ and $T \Delta S$ term

material), methanol is more hydrophilic than acetone [50]. This is reflected by the negative value of $\Delta A_{\mathrm{m}}\left(\mathrm{A}_{\mathrm{q}}\right)=$ $-19.5 \mathrm{~kJ} \mathrm{~mol}^{-1}$ in Table 2 , which is the result of an energetic contribution of $-6.3 \mathrm{~kJ} \mathrm{~mol}^{-1}$ and an entropic contribution of $13.2 \mathrm{~kJ} \mathrm{~mol}^{-1}$. Note that the intermolecular interaction energies amount to zero, such that no gas-phase corrections are needed. Comparing the values of $\Delta E$ and $\Delta E_{\mathrm{ls}}$ or $T \Delta S$ and $T \Delta S_{\mathrm{ls}}$ from approach I (Eq. 11) allows us to quantify the surrounding-surrounding contribution to the energy and entropy of the acetone to methanol mutation. $\Delta E$ is built up from $-21.6 \mathrm{~kJ} \mathrm{~mol}^{-1}\left(\Delta E_{\mathrm{ls}}\right)$ as a result of stronger interactions between methanol and the water molecules and a loss of $15.3 \mathrm{~kJ} \mathrm{~mol}^{-1}\left(\Delta E_{\mathrm{ss}}\right)$ due to reduced solvent-solvent interactions between these water molecules. The favourable entropic contribution of $13.2 \mathrm{~kJ} \mathrm{~mol}^{-1}$ predominantly stems from the solvent- 
solvent reorganisation of $T \Delta S_{\mathrm{ss}}=15.3 \mathrm{~kJ} \mathrm{~mol}^{-1}$ exactly cancelling the unfavourable energy contribution of $\Delta E_{\mathrm{ss}}$. What remains is a slightly unfavourable contribution of the ligand-surrounding entropy, $T \Delta S_{\mathrm{ls}}=-2.1 \mathrm{~kJ} \mathrm{~mol}^{-1}$, probably due to the smaller, more spherical size of the solute.

In the hydrophobic $\mathrm{C}_{\mathrm{APO}}$ cavity, the free energy associated with the same mutation is unfavourable by $11.7 \mathrm{~kJ}$ $\mathrm{mol}^{-1}$, due to an unfavourable $\Delta E_{\mathrm{ls}}=22.2 \mathrm{~kJ} \mathrm{~mol}^{-1}$ resulting from incomplete 'solvation' in a cavity that is too large for the methanol molecule, partly compensated by an increased ligand entropy. In the $\mathrm{C}_{\mathrm{HB}}$ cavity with a smaller volume, the energy change is small $\Delta E_{\mathrm{ls}}=-1.1 \mathrm{~kJ}$ $\mathrm{mol}^{-1}$, while the increase in ligand-surrounding entropy $T \Delta S_{\mathrm{ls}}$ is of comparable size $\left(11-14 \mathrm{~kJ} \mathrm{~mol}^{-1}\right)$, indicating that more relevant configurations are accessible for methanol than for acetone in both cavities.

Considering in more detail the relative binding free energy, $\Delta \Delta A_{\mathrm{b}}$ of acetone and methanol in $\mathrm{C}_{\mathrm{HB}}$, we obtain a moderate value of $4.4 \mathrm{~kJ} \mathrm{~mol}^{-1}$, which is built up from a small unfavourable energetic contribution $\Delta \Delta E=1.8$ $\mathrm{kJ} \mathrm{mol}^{-1}$ and a small unfavourable entropic contribution $T \Delta \Delta S=-2.5 \mathrm{~kJ} \mathrm{~mol}^{-1}$. It may be tempting to conclude from these numbers that the binding of the two compounds is governed by the same principles. However, the values of $\Delta \Delta E$ and $T \Delta \Delta S$ are obscured by a large, exactly compensating value of $\Delta \Delta E_{\mathrm{ss}}=T \Delta \Delta S_{\mathrm{ss}}=-18.6 \mathrm{~kJ} \mathrm{~mol}^{-1}$. Considering the reduced terms, which leave out the surrounding-surrounding energies and entropies, we see that $\Delta \Delta A_{\mathrm{b}}$ is built up from a large $\Delta \Delta E_{\mathrm{ls}}=20.5 \mathrm{~kJ} \mathrm{~mol}^{-1}$ (as the result of a significantly larger desolvation energy of methanol than of acetone) and a considerable $T \Delta \Delta S_{\mathrm{ls}}=$ $16.1 \mathrm{~kJ} \mathrm{~mol}^{-1}$ (as the results of methanol having more space to move around the small cavity than acetone). So the small value of $\Delta \Delta A_{\mathrm{b}}$ is the result of two distinct molecular features in which the two molecules differ. The above example nicely demonstrates how the surroundingsurrounding energy and entropy, which do not contribute to $\Delta \Delta A_{\mathrm{b}}$, may obscure a molecular interpretation of basic thermodynamic properties. Similar considerations may very well explain the observation of Biela et al. [25] where very similar thermodynamic profiles were obtained for two ligands with distinct binding poses.

The values for $\Delta E_{\mathrm{ss}}$ and $T \Delta S_{\mathrm{ss}}$ range from -3.4 to $15.3 \mathrm{~kJ} \mathrm{~mol}^{-1}$ and the corresponding relative surroundingsurrounding binding energies $\left(\Delta \Delta E_{\mathrm{ss}}\right)$ and entropies $\left(T \Delta \Delta S_{\mathrm{ss}}\right)$ amount to $-7.8\left(\mathrm{C}_{\mathrm{APO}}\right)$ and $-18.6 \mathrm{~kJ} \mathrm{~mol}^{-1}\left(\mathrm{C}_{\mathrm{HB}}\right)$, respectively. Not unexpected for the host model molecules completely shielding the ligand from direct interactions with the solvent, the surrounding-surrounding energy entropy compensation is smaller in the $\mathrm{C}_{\mathrm{APO}}$ and $\mathrm{C}_{\mathrm{HB}}$ systems than free in solution, leading to negative values for $\Delta \Delta E_{\mathrm{ss}}$. This suggests that the more readily converging reduced terms cannot straightforwardly be used as a replacement for the full energetic and entropic terms and that the surrounding-surrounding contributions do depend strongly on the actual surrounding of the ligand and cannot be expected to cancel in the relative values. The fact that $\Delta \Delta E_{\mathrm{ss}}$ and $T \Delta \Delta S_{\mathrm{ss}}$ are so different in the two host systems also shows that they should really be excluded from the interpretation of free energy differences in which they cancel. Inclusion of the surroundingsurrounding terms will obscure differences between the hosts while the reduced terms offer physical interpretations more relevant for drug design.

The reduced terms do not correspond to experimentally observable quantities and as such cannot be validated by experimental means. The decomposition of the energetic and entropic contributions in terms of a ligand and its surroundings is intuitive, but different choices can be made including fewer or more terms that are compensated in $\Delta E$ and $T \Delta S$. The observation that $\Delta E$ and $T \Delta S$ contain exactly compensating terms allows one to argue that, even though not corresponding to experimental observations, the reduced $\Delta E_{\mathrm{ls}}$ and $T \Delta S_{\mathrm{ls}}$ terms may be of more use in computational drug design than their full counterparts. After all, what use is an optimisation in terms of energy if a significant portion of it is compensated by a loss in entropy and the overall affinity is not improved?

More importantly, many of the optimisations either try to rigidify the ligand or address an additional ligand-surrounding interaction, which will be more easily quantified in terms of the well-converging reduced terms. Therefore, it may be advisable and also feasible to first characterise a lead compound and its affinity in terms of $\Delta E_{\mathrm{ls}}$ and $T \Delta S_{\mathrm{ls}}$ and to rationally optimise these terms in silico in order to design a new compound with a higher affinity. Whether part of the full energy is subsequently compensated by the full entropy is irrelevant for the binding affinity.

\section{Conclusion}

The free energy difference between acetone and methanol in solution and when bound to two model host systems was calculated. Three approaches were taken to quantify the energetic and entropic contributions to the free energies. Moreover, these were described in terms of ligand-surrounding energies and entropies, effectively also quantifying the (exactly compensating) surrounding-surrounding energies and entropies. Internal consistency of the calculations was ensured by investigating multiple cycle closures for the state functions. The convergence of all thermodynamic properties was monitored.

The first approach, calculating the entropy as a difference between the free energy and the energy leads to the smallest statistical uncertainties for this highly simplified host model system. Quantifying the entropy from the 
temperature dependence of the free energy in the second approach leads to comparable values, but a proper propagation of the error estimates increases the statistical uncertainty significantly. The third approach, in which the entropy is directly estimated from thermodynamic integration, does not lead to converged results on the timescales investigated here. This does not hold for the reduced thermodynamic terms $\left(\Delta E_{\mathrm{ls}}\right.$ and $\left.T \Delta S_{\mathrm{ls}}\right)$, for which the first and third approaches yield comparable estimates, except for a contribution due to modified bond-length constraints.

Although not corresponding to experimentally accessible quantities, the reduced terms can be readily calculated from molecular simulations and may prove very powerful in the thermodynamic optimisation of lead compounds in computational drug design, as the intrinsic energy-entropy compensation due to the surrounding is not included. We have described examples of how the surrounding-surrounding energy-entropy compensation obscures a proper molecular interpretation of the thermodynamic terms. Rather, we propose to use the reduced terms, opening the way to new design strategies.

Acknowledgments Financial support from Grant No. LS08-QM3 of the Vienna Science and Technology Fund (WWTF), Grant No. 260408 of the European Research Council (ERC) and the PhD programme "BioToP-Biomolecular Technology of Proteins" (Austrian Science Funds, FWF Project W1224) is gratefully acknowledged.

Open Access This article is distributed under the terms of the Creative Commons Attribution License which permits any use, distribution, and reproduction in any medium, provided the original author(s) and the source are credited.

\section{References}

1. Ladbury JE, Klebe G, Freire E (2010) Adding calorimetric data to decision making in lead discovery: a hot tip. Nat Rev Drug Discov 1:23-27. doi:10.1038/nrd3054

2. Klebe G (2006) Virtual ligand screening: strategies, perspectives and limitations. Drug Discov Today 13-14:580-594. doi:10. 1016/j.drudis.2006.05.012

3. Leavitt S, Freire E (2001) Direct measurement of protein binding energetics by isothermal titration calorimetry. Curr Opin Struct Biol 5:560-566

4. Lafont V, Armstrong AA, Ohtaka H, Kiso Y, Mario Amzel L, Freire E (2007) Compensating enthalpic and entropic changes hinder binding affinity optimization. Chem Biol Drug Des 6:413-422. doi:10.1111/j.1747-0285.2007.00519.x

5. Freire E (2008) Do enthalpy and entropy distinguish first in class from best in class? Drug Discov Today 19-20:869-874. doi: 10.1016/j.drudis.2008.07.005

6. Freire E (2009) A thermodynamic approach to the affinity optimization of drug candidates. Chem Biol Drug Des 5:468-472. doi:10.1111/j.1747-0285.2009.00880.x

7. Falconer RJ, Collins BM (2011) Survey of the year 2009: applications of isothermal titration calorimetry. J Mol Recogn 1:1-16. doi:10.1002/jmr.1073
8. Peter C, Oostenbrink C, van Dorp A, van Gunsteren WF (2004) Estimating entropies from molecular dynamics simulations. J Chem Phys 6:2652-2661. doi:10.1063/1.1636153

9. Reinhardt WP, Miller MA, Amon LM (2001) Why is it so difficult to simulate entropies, free energies, and their differences? Acc Chem Res 7:607-614

10. Skillman AG (2012) SAMPL3: blinded prediction of host-guest binding affinities, hydration free energies, and trypsin inhibitors. J Comput Aided Mol Des 5:473-474. doi:10.1007/s10822012-9580-Z

11. Muddana HS, Daniel Varnado C, Bielawski CW, Urbach AR, Isaacs L, Geballe MT et al (2012) Blind prediction of host-guest binding affinities: a new SAMPL3 challenge. J Comput Aided Mol Des 5:475-487. doi:10.1007/s10822-012-9554-1

12. Oostenbrink C (2009) Efficient free energy calculations on small molecule host-guest systems - a combined linear interaction energy/ one-step perturbation approach. J Comput Chem 2:212-221. doi: $10.1002 /$ jcc. 21116

13. Schlitter J (1993) Estimation of absolute and relative entropies of macromolecules using the covariance matrix. Chem Phys Lett 6:617-621. doi:10.1016/0009-2614(93)89366-P

14. Karplus M, Kushick JN (1981) Method for estimating the configurational entropy of macromolecules. Macromolecules 2:325-332. doi:10.1021/ma50003a019

15. Hensen U, Grubmüller H, Lange OF (2009) Adaptive anisotropic kernels for nonparametric estimation of absolute configurational entropies in high-dimensional configuration spaces. Phys Rev E 1:011913

16. Hensen U, Lange OF, Grubmuller H (2010) Estimating absolute configurational entropies of macromolecules: the minimally coupled subspace approach. PLoS ONE 2:e9179. doi:10.1371/journal. pone.0009179

17. Harpole KW, Sharp KA (2011) Calculation of configurational entropy with a Boltzmann-quasiharmonic model: the origin of high-affinity protein-ligand binding. J Phys Chem B 30:94619472. doi:10.1021/jp111176x

18. Dolenc J, Baron R, Oostenbrink C, Koller J, van Gunsteren WF (2006) Configurational entropy change of netropsin and distamycin upon DNA minor-groove binding. Biophys J 4:1460-1470. doi:10.1529/biophysj.105.074617

19. Lange JH, Venhorst J, van Dongen MJ, Frankena J, Bassissi F, de Bruin NM et al (2011) Biophysical and physicochemical methods differentiate highly ligand-efficient human D-amino acid oxidase inhibitors. Eur J Med Chem 10:4808-4819. doi:10.1016/j.ejmech. 2011.04.023

20. Baron R, Setny P, Andrew McCammon J (2010) Water in cavity-ligand recognition. J Am Chem Soc 34:12091-12097. doi: 10.1021/ja1050082

21. DeLorbe JE, Clements JH, Teresk MG, Benfield AP, Plake HR, Millspaugh LE et al (2009) Thermodynamic and structural effects of conformational constraints in protein-ligand interactions. Entropic paradoxy associated with ligand preorganization. J Am Chem Soc 46:16758-16770. doi:10.1021/ja904698q

22. Ben-Naim A, Marcus Y (1984) Solvation thermodynamics of nonionic solutes. J Chem Phys 4:2016-2027

23. van der Vegt NFA, van Gunsteren WF (2004) Entropic contributions in cosolvent binding to hydrophobic solutes in water. J Phys Chem B 3:1056-1064. doi:10.1021/jp030532c

24. Ozal TA, van der Vegt NF (2006) Confusing cause and effect: energy-entropy compensation in the preferential solvation of a nonpolar solute in dimethyl sulfoxide/water mixtures. J Phys Chem B 24:12104-12112. doi:10.1021/jp061608i

25. Biela A, Sielaff F, Terwesten F, Heine A, Steinmetzer T, Klebe G (2012) Ligand binding stepwise disrupts water network in thrombin: enthalpic and entropic changes reveal classical hydrophobic effect. J Med Chem. doi:10.1021/jm300337q 
26. Kirkwood JG (1935) Statistical mechanics of fluid mixtures. J Chem Phys 5:300-313. doi:10.1063/1.1749657

27. McQuarrie DA (2000) Statistical mechanics, 1st edn. University Science Book, Mill Valley

28. Hermans J, Shankar S (1986) The free-energy of xenon binding to myoglobin from molecular-dynamics simulation. Isr J Chem 2:225-227

29. Buch I, Giorgino T, De Fabritiis G (2011) Complete reconstruction of an enzyme-inhibitor binding process by molecular dynamics simulations. Proc Natl Acad Sci USA 25:10184-10189. doi:10.1073/pnas.1103547108

30. Boresch S, Tettinger F, Leitgeb M, Karplus M (2003) Absolute binding free energies: a quantitative approach for their calculation. J Phys Chem B 35:9535-9551. doi:10.1021/jp0217839

31. Roux B, Nina M, Pomès R, Smith JC (1996) Thermodynamic stability of water molecules in the bacteriorhodopsin proton channel: a molecular dynamics free energy perturbation study. Biophys J 2:670-681. doi:10.1016/S0006-3495(96)79267-6

32. Woo HJ, Roux B (2005) Calculation of absolute protein-ligand binding free energy from computer simulations. Proc Natl Acad Sci USA 19:6825-6830. doi:10.1073/pnas.0409005102

33. Berendsen H, Postma J, van Gunsteren W, Hermans J (1981) In: Pullman B (ed) Intermolecular forces D. Reidel Publishing Company, Dordrecht

34. Schmid N, Christ CD, Christen M, Eichenberger AP, van Gunsteren WF (2012) Architecture, implementation and parallelisation of the GROMOS software for biomolecular simulation. Comput Phys Commun 4:890-903. doi:10.1016/j.cpc.2011.12.014

35. Schmid N, Eichenberger A, Choutko A, Riniker S, Winger M, Mark A et al (2011) Definition and testing of the GROMOS force-field versions 54A7 and 54B7. Eur Biophys J 7:843-856. doi:10.1007/s00249-011-0700-9

36. Berendsen HJC, Postma JPM, van Gunsteren WF, DiNola A, Haak JR (1984) Molecular dynamics with coupling to an external bath. J Chem Phys 8:3684-3690

37. Berendsen HJC (2007) Simulating the physical world. Cambridge University Press, Cambridge, MA

38. Hockney RW, Eastwood JW (1992) Computer simulation using particles. Institute of Physics

39. Ryckaert J, Ciccotti G, Berendsen HJC (1977) Numerical integration of the Cartesian equations of motion of a system with constraints: molecular dynamics of n-alkanes. J Comput Phys 3:327-341. doi:10.1016/0021-9991(77)90098-5
40. Beutler TC, Mark AE, van Schaik RC, Gerber PR, van Gunsteren WF (1994) Avoiding singularities and numerical instabilities in free energy calculations based on molecular simulations. Chem Phys Lett 6:529-539. doi:10.1016/0009-2614(94)00397-1

41. Heinz TN, Hünenberger PH (2004) A fast pairlist-construction algorithm for molecular simulations under periodic boundary conditions. J Comput Chem 12:1474-1486. doi:10.1002/jcc. 20071

42. Tironi IG, Sperb R, Smith PE, van Gunsteren WF (1995) A generalized reaction field method for molecular dynamics simulations. J Chem Phys 13:5451-5459. doi:10.1063/1.469273

43. Heinz TN, van Gunsteren WF, Hünenberger PH (2001) Comparison of four methods to compute the dielectric permittivity of liquids from molecular dynamics simulations. J Chem Phys 3:1125-1136. doi:10.1063/1.1379764

44. Riniker S, Christ CD, Hansen HS, Hünenberger PH, Oostenbrink C, Steiner D et al (2011) Calculation of relative free energies for ligand-protein binding, solvation, and conformational transitions using the GROMOS software. J Phys Chem B 46:13570-13577. doi:10.1021/jp204303a

45. Allen MP, Tildesley DJ (1989) Computer simulation of liquids. Clarendon Press, Oxford

46. Berendsen HJC (2011) A student's guide to data and error analysis. Cambridge University Press, Cambridge

47. Carlsson J, Aqvist J (2006) Calculations of solute and solvent entropies from molecular dynamics simulations. Phys Chem Chem Phys 46:5385-5395. doi:10.1039/b608486a

48. Carlsson J, Aqvist J (2009) Absolute hydration entropies of alkali metal ions from molecular dynamics simulations. J Phys Chem B 30:10255-10260. doi:10.1021/jp900818z

49. van Gunsteren WF, Beutler TC, Fraternali F, King PM, Mark AE, Smith PE (1993) In: van Gunsteren WF, Weiner PK, Wilkinson $\mathrm{AJ}$ (eds) Computer simulation of biomolecular systems, theoretical and experimental applications. Escom Science Publishers, Leiden

50. Horta BAC, Fuchs PFJ, van Gunsteren WF, Hünenberger PH (2011) New interaction parameters for oxygen compounds in the GROMOS force field: improved pure-liquid and solvation properties for alcohols, ethers, aldehydes, ketones, carboxylic acids, and esters. J Chem Theory Comput 4:1016-1031. doi:10.1021/ ct1006407 